\title{
Identification of the Eutrema salsugineum ESMYB90 gene important for anthocyanin biosynthesis
}

\author{
Yuting $\mathrm{Qi}^{1+}$, Caihong $\mathrm{Gu}^{1 \dagger}$, Xingjun Wang ${ }^{1,2}$, Shiqing $\mathrm{Gao}^{3}$, Changsheng $\mathrm{Li}^{2}$, Chuanzhi Zhao ${ }^{1,2}$, Chuanshun $\mathrm{Li}^{1}$, \\ Changle $\mathrm{Ma}^{1}$ and Quan Zhang ${ }^{1 *}$
}

\begin{abstract}
Background: Anthocyanins contribute to coloration and antioxidation effects in different plant tissues. MYB transcription factors have been demonstrated to be a key regulator for anthocyanin synthesis in many plants. However, little information was available about the MYB genes in the halophyte species Eutrema salsugineum.

Result: Here we report the identification of an important anthocyanin biosynthesis regulator EsMYB90 from Eutrema salsugineum, which is a halophyte tolerant to multiple abiotic stresses. Our phylogenetic and localization analyses supported that EsMYB90 is an R2R3 type of MYB transcription factor. Ectopic expression of EsMYB90 in tobacco and Arabidopsis enhanced pigmentation and anthocyanin accumulation in various organs. The transcriptome analysis revealed that 42 genes upregulated by EsMYB90 in 35S:EsMYB90 tobacco transgenic plants are required for anthocyanin biosynthesis. Moreover, our qRT-PCR results showed that EsMYB90 promoted expression of early (PAL, CHS, and CHI) and late (DFR, ANS, and UFGT) anthocyanin biosynthesis genes in stems, leaves, and flowers of 35S:ESMYB90 tobacco transgenic plants.

Conclusions: Our results indicated that ESMYB90 is a MYB transcription factor, which regulates anthocyanin biosynthesis genes to control anthocyanin biosynthesis. Our work provides a new tool to enhance anthocyanin production in various plants.
\end{abstract}

Keywords: Anthocyanin, Flavonoid, Eutrema salsugineum, R2R3 MYB transcription factor, EsMYB90, Transcriptional regulation, Anthocyanin biosynthesis genes

\section{Background}

Flavonoids which are derivatives of the phenylpropanoid/ flavonoid pathway mainly contain proanthocyanidins (PAs), anthocyanins and flavonols [1-3]. As important pigments, anthocyanins are responsible for red, purple, violet and blue colors in flowers, fruits, and leaves, which determine economic traits of crops and ornamental plants

\footnotetext{
* Correspondence: zhangquan@sdnu.edu.cn

'Yuting Qi and Caihong Gu contributed equally to this work and are parallel/ joint first authors.

'Shandong Provincial Key Laboratory of Plant Stress Research, College of Life Science, Shandong Normal University, Jinan 250014, China

Full list of author information is available at the end of the article
}

[4-7]. Anthocyanins are the end products of a specific branch in the phenylpropanoid/flavonoid biosynthesis pathway. Enzymes involved in anthocyanin biosynthesis have been extensively studied in many plant species [8]. Catalyzed by phenylalanine ammonia-lyase (PAL), the initial step of the flavonoid pathway is the conversion of phenylalanine into trans-cinnamic acid [9], while chalcone synthase (CHS) catalyzes the first committed step in the flavonoid biosynthesis to form naringenin chalcone. Chalcone isomerase $(\mathrm{CHI})$ cyclizes chalcone to form naringenin [8]. The naringenin is then converted into dihydrokaempferol (DHK) by flavanone $3 \beta$-hydroxylase

C C The Author(s). 2020 Open Access This article is licensed under a Creative Commons Attribution 4.0 International License, which permits use, sharing, adaptation, distribution and reproduction in any medium or format, as long as you give appropriate credit to the original author(s) and the source, provide a link to the Creative Commons licence, and indicate if changes were made. The images or other third party material in this article are included in the article's Creative Commons licence, unless indicated otherwise in a credit line to the material. If material is not included in the article's Creative Commons licence and your intended use is not permitted by statutory regulation or exceeds the permitted use, you will need to obtain permission directly from the copyright holder. To view a copy of this licence, visit http://creativecommons.org/licenses/by/4.0/ The Creative Commons Public Domain Dedication waiver (http://creativecommons.org/publicdomain/zero/1.0/) applies to the data made available in this article, unless otherwise stated in a credit line to the data. 
(F3H). DHK is further hydroxylated to dihydroquercetin (DHQ) by flavonoid 3'-hydroxylase (F3'H), or to dihydromyricetin (DHM) by flavonoid 3',5'-hydroxylase (F3'5'H). Dihydroflavonol 4-reductase (DFR) converts DHQ into leucocyanidin, which is further converted into anthocyanidins by anthocyanidin synthase (ANS). Finally, UDP-glucose: flavonoid 3-O-glucosyltranferase (UFGT) catalyzes glycosylation of anthocyanidins to form anthocyanins [8, 10-12].

MYB transcription factors play a central role in regulating expression of genes encoding major enzymes for anthocyanin biosynthesis via forming the transcriptional complex containing MYB-bHLH-WD40 (MBW) [1, 13-15]. Expression of early biosynthesis genes (EBGs) such as CHS and $C H I$, is regulated by MYB11, MYB12 and MYB111, whereas PAP1 to PAP4 (AtMyb75, AtMyb90, AtMyb113, and $A t$ Myb114) control expression of late biosynthesis genes (LBGs) including DFR, ANS, and UFGT in Arabidopsis [16, 17]. In Arabidopsis, up-regulation of one of MYB75, MYB90, MYB113 and MYB114 genes is sufficient to increase anthocyanin accumulation in young leaves $[15,17]$. For example, the well-known Arabidopsis AtMYB75 (PAP1) gene directs anthocyanin production in leaves, roots, flowers, and fruits $[18,19]$. Overexpression of AtMyb75 in Arabidopsis and tobacco results in upregulation of PAL, CHS and DFR genes [19, 20]. Similarly, AtMYB75 induces anthocyanin production in tomato (Solanum lycopersicum L.) via promoting the DFR expression [18]. Furthermore, the sequence variation of AtMYB9O (PAP2) is causal for natural variation in anthocyanin accumulation [17, 21]. AtMYB90 may act together with TTG1 (a WD40 protein) and different bHLH partners including TT8, GL3 or EGL3 [15, 18, 22]. Moreover, in Arabidopsis the ternary complexes formed by R2R3-MYB, bHLH and the WD repeat protein activate the biosynthetic genes required for proanthocyanidin accumulation in the innermost cell layer of the seed coat [23]. The R2R3 MYB protein TT2 (MYB123) is also a key regulator of proanthocyanidin accumulation in developing seeds [24].

MYB transcription factors are involved in regulation of anthocyanin synthesis in many plants, such as Arabidopsis [15, 17, 24, 25], cauliflower (Brassica oleracea var botrytis) [26], bok choy (Brassica rapa var. chinensis) [27], apple (Malus $\times$ domestica) [11, 28-30], peach (Prunus persica) [14, 31], pear (Pyrus pyrifolia) [13, 32, 33],strawberry (Fragaria $x$ ananassa) [34], snapdragon (Antirrhinum majus) [35], Chrysanthemum [10], grape hyacinth (Muscari armeniacum) [36], grapevine (Vitis vinifera) $[37,38]$, chinese bayberry (Myrica rubra) $[6,39]$, Epimedium sagittatum [40, 41], poplar (Populus spp) [42] and potato (Solanum tuberosum L) [43]. In addition, some $M Y B$ genes are up-regulated under various stress conditions $[15,17]$. However, the transcriptional regulation of anthocyanin synthesis by MYB in stress-tolerant plants is not well studied. Eutrema salsugineum (salt cress), a stress-tolerant model halophyte, is highly tolerant to cold, salt, drought, oxidative stress, and nitrogen deficiency. In Eutrema salsugineum, many stress-tolerant related genes, such as SOS1, HKT1, and nsLTP4, have been identified [44-49]. By analyzing the differentially expressed regulatory genes between Arabidopsis and E.salsugineum, it was found that the regulatory functions of 307 transcription factors in 50 different families were significantly different [50]. Another study found that EsMYB96/WAX1 from E.salsugineum under the RD29A promoter improved drought tolerance with increased accumulation of cuticular wax and ascorbic acid in transgenic Arabidopsis [51]. So far, there is no research report on the anthocyanin synthesis of EsMYBs in E.salsugineum. Here, we reported our functional analysis of the MYB transcription factor EsMYB90 in anthocyanin synthesis. Our phylogenetic and localization analyses suggest that EsMYB90 is an R2R3 type of MYB transcriptional factor. Ectopic expression of EsMYB90 in tobacco and Arabidopsis led to significantly increased pigmentation and production of anthocyanins in leaves, stems, and flowers. Our further RNA-seq and qRT-PCR analyses showed that EsMYB90 promoted expression of anthocyanin early biosynthesis genes (EBGs: NtCHS, $N t C H I$, and $N t F 3 H$ ) and late biosynthesis genes (LBGs: NtDFR, NtANS, and NtUFGT) in 35S:EsMYB90 tobacco transgenic plants. Our study identified a MYB transcription factor, which plays an important role in plant anthocyanin biosynthesis.

\section{Results}

Database mining identifies EsMYB90, a candidate regulator for anthocyanin synthesis

Eutrema salsugineum is a stress-tolerance halophyte, which produces purple flower buds after vernalization [52]. Since $M Y B$ genes are required for anthocyanin synthesis [13, 15, 36], we identified which $M Y B$ controls this purple phenotype in E. salsugineum. After comparing MYB genes obtained from the transcriptome of E. salsugineum based on our previously published results [46], with $72 M Y B$ genes known acting as proanthocyanin (PA) and anthocyanin regulators in other plants, we found one candidate $M Y B$ gene, named as EsMYB90.

To determine the relationship of EsMYB90 to characterized flavonoid and PA MYBs, we performed similarity analysis at the protein level. Our results showed that EsMYB90 has 80.5, 78.9, 78.4, 74.4, 69.4, 65.9, 50\% identities respectively to 7 MYB proteins, i.e. BoMYB1, AtMYB90, BrMYB114, AtMYB75, CrMYB114, AtMYB11 3, and AtMYB114 (Fig. 1a). In addition, similarities between EsMYB90 and other 10 MYB proteins range from 44.1 to $39.0 \%$ (Fig. 1a). Those MYB proteins with high similarities to EsMYB90 belong to the class of R2R3-MYB, which have a conserved DNA-binding 


\section{A}

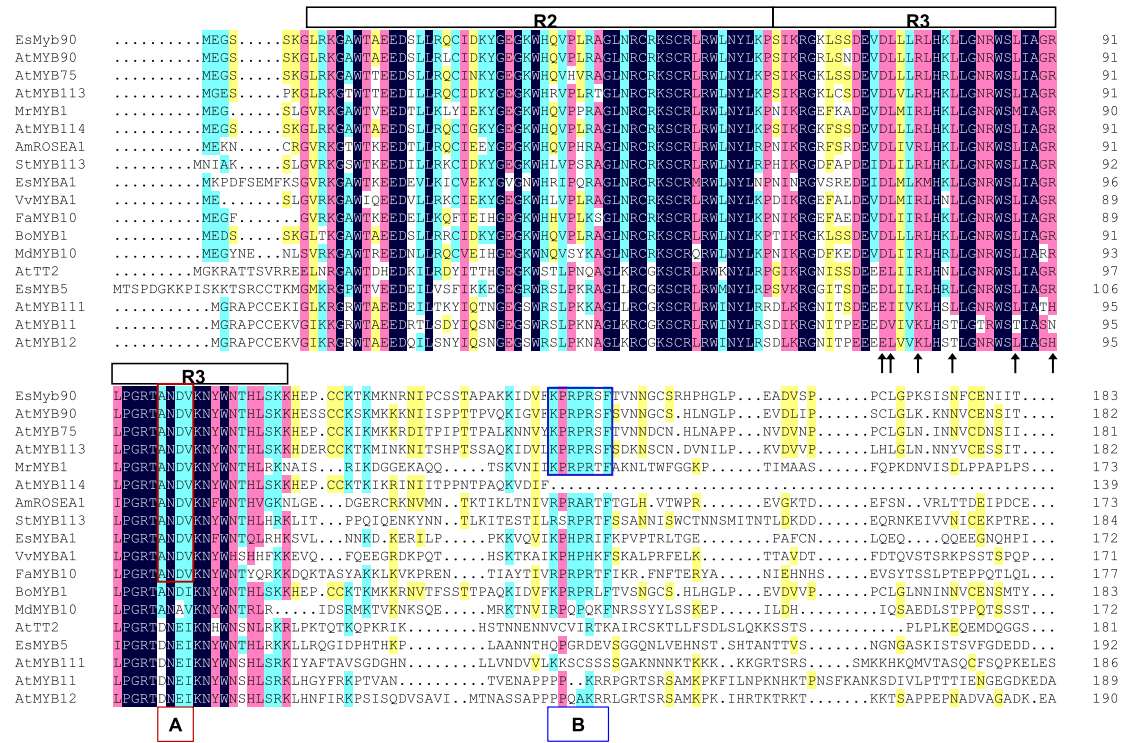

B

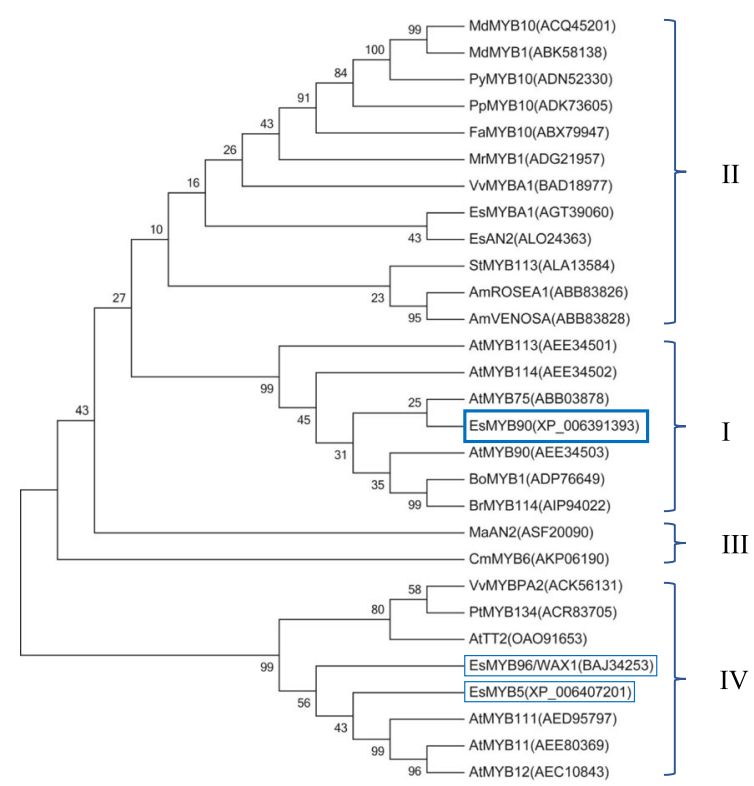

Fig. 1 Alignment and phylogenetic analysis of EsMYB90 and other MYB proteins. a The EsMYB90 protein sequence was aligned with a set of related R2R3-MYB proteins from 10 plant species. Identical amino acids are shaded in dark blue, and the greater than or equal to the identity of 75,50 , and $33 \%$ are indicated in shade of pink, light blue, and yellow, respectively. R2 and R3 domains refer to two repeats of the MYB DNA binding domain. Box (A): the conserved motif ANDV in the R3 domain for dicot anthocyanin-promoting MYBs; Box (B): the C-terminal-conserved motif KPRPR [S/T] F for Arabidopsis anthocyanin-promoting MYBs; Black arrows: the specific residues of [D/E]L $x_{2}[R / K] x_{3} L x_{6} L x_{3} R$ that confer to the interaction with bHLH. b Phylogenetic analysis of ESMYB90 and other 28 MYB proteins. The MYB protein sequences were downloaded from the GenBank database with accession numbers showed in the diagram. EsMYB5, EsMYB90 and EsMYB96 are from E. salsugineum, and EsMYB90 is highlighted in a bold blue box, while EsMYB5 and EsMYB96 are highlighted in a thin blue box. MYB proteins from the cruciferae plants in the clade I have higher identities to EsMYB90 than that in clades II to IV

domain ( $\mathrm{R} 2$ and $\mathrm{R} 3$ repeats) in the $\mathrm{N}$-terminal and a variable C-terminal region [41, 53, 54]. The ANDV motif (marked by red A box in Fig. 1a), a characteristic identifier for anthocyanin-promoting MYBs in dicots [10], existed in EsMYB90, AtMYB90, AtMYB75, AtMYB113, AtMYB114, AmROSEA1, StMYB113, EsMYBA1(AGT39060), VvMYBA1,
MrMYB1, and FaMYB10, while the C-terminal-conserved motif KPRPR [S/T] F for Arabidopsis anthocyanin-promoting MYBs [25, 36] (marked by blue B box in Fig. 1a), was only found in EsMYB90, AtMYB75, AtMYB90, AtMYB113, and MrMYB1. Moreover, EsMyb90 has a conserved [D/E]Lx2[R/ K]x3Lx6Lx3R motif (marked by black arrows in Fig. 1a), 
which is required for interaction with R/B-like bHLH proteins $[10,16]$.

To further identify the relationship of EsMYB90 to other MYB proteins, we generated a phylogenetic tree with 29 MYB proteins involved in anthocyanin synthesis in 16 plants. Our results demonstrated that EsMYB90 was clustered in the clade I (Fig. 1b), which consists of AtMYB75, AtMYB90, AtMYB113, AtMYB114, BoMYB1 and BrMYB114 that are important for anthocyanin accumulation [17, 19, 25-27, 55]. EsMYB90 has a relatively farer phylogenetic relationship to MYB proteins in clades II, III, and IV, although those MYBs promote biosynthesis of PA and anthocyanin, except for EsMYB5(XP_006407201) have no research reports yet.

In summary, our results suggest that EsMYB90 is a R2R3-MYB, which may function in proanthocyanin and anthocyanin synthesis.

\section{Expression pattern of EsMYB90 in E.salsugineum and subcellular localization of the protein}

In order to detect the expression pattern of EsMYB90, we collected leaves, petioles, stems, roots and flowers of E.salsugineum, and performed qRTPCR. Our result showed that EsMYB90 was expressed in all examined tissues of E.salsugineum, among which it has the highest expression level in petiole (Fig. 2b), followed by stems and flowers (Fig. 2c,d), but a relative lower expression in leaves and roots (Fig. 2a,e). This result is consistent with the color phenotype of different tissues observed, suggesting the expression of EsMYB90 is related to the synthesis of anthocyanins (Fig. 2a-f).

To test the subcellular localization of EsMYB90, we examined the transient expression of YFP-EsMYB90 fusion protein in onion epidermal cells. Our results showed that YFP signals were observed in both cytoplasm and nucleus of the onion epidermal cells expressing 35S:YFP (Fig. 2g-i), while the YFP signal was only detected in the nucleus in cells expressing 35S: YFP-EsMYB90 (Fig. 2j-1). Our result showed EsMYB90 is localized to the nucleus, suggesting that as other MYB proteins EsMYB90 also functions as a transcriptional regulator.

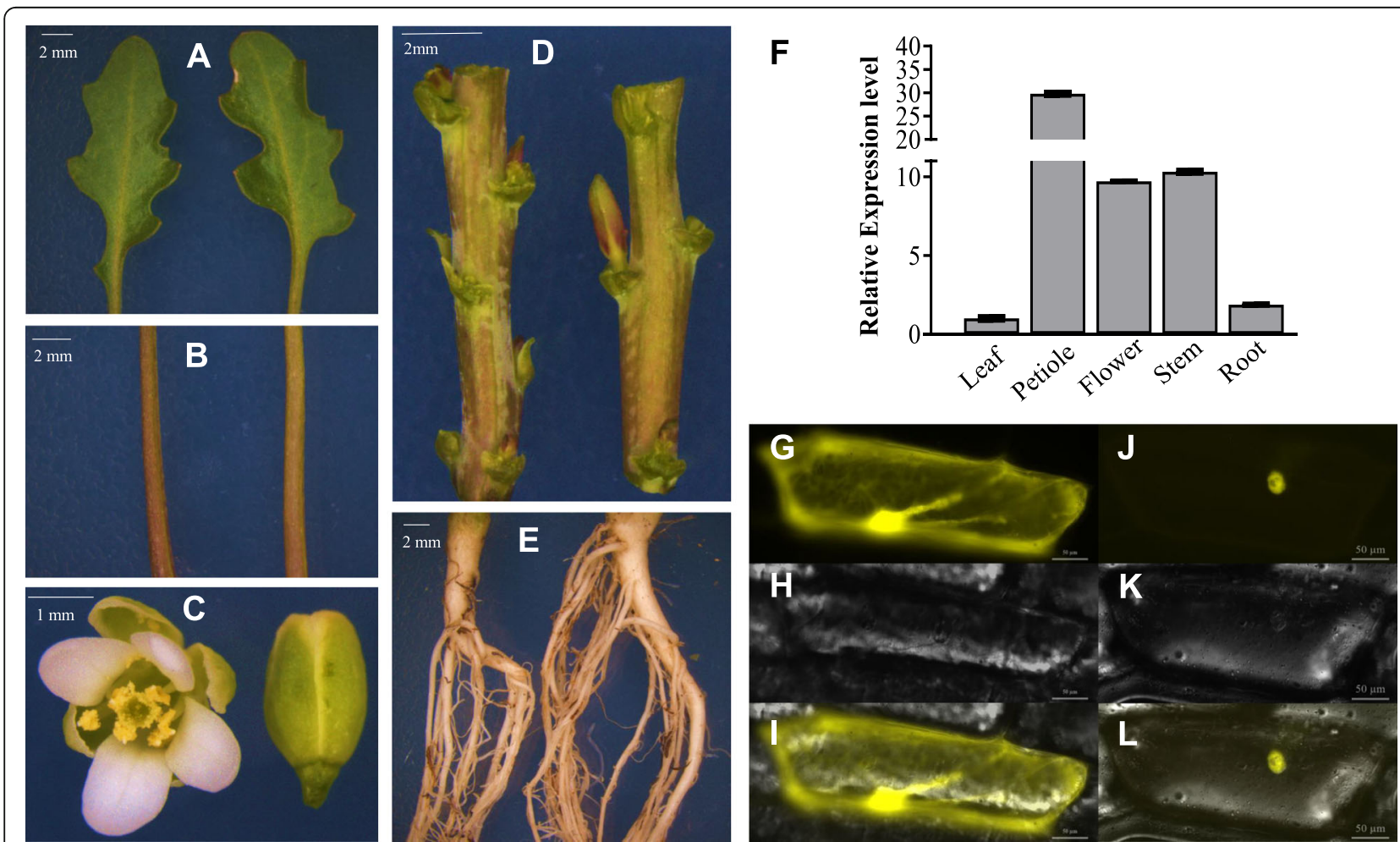

Fig. 2 Expression pattern of EsMYB90 in E. salsugineum and subcellular localization of the protein. The leaves (a), petioles (b), flowers (c), stems (d), and roots (e) from thirty-five-week-old E.salsugineum plants were collected for phenotypic observation. $\mathbf{f}$ The expression levels of EsMyb90 in leaves, petioles, flowers, stems, and roots from thirty-five-week-old E. salsugineum, and vertical bars indicate standard errors of the 3 biological replicates. Transient expression of the 35S:YFP-ESMYB90 in onion epidermal cells showing EsMYB90 is localized in the nucleus. $\mathbf{g}$ An onion epidermal cell expressing 35S:YFP showing YFP signals in both cytoplasm and nucleus; (h) An onion epidermal cell expressing 35S:YFP in the bright field; (i) The merged image of $\mathrm{G}$ and $\mathrm{H}$; (j) An onion epidermal cell expressing 35S:YFP-ESMYB90 exhibiting the YFP signal only in the nucleus; (k)

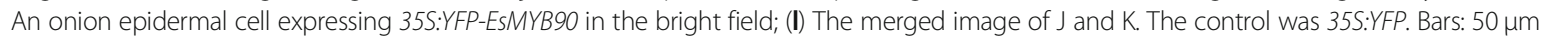


Ectopic expression of EsMYB90 promotes anthocyanin accumulation in tobacco and Arabidopsis

To investigate the possible function of EsMYB90 in anthocyanin biosynthesis, we generated the 35S: EsMYB90 construct to ectopically expression EsMYB90 in tobacco and Arabidopsis (Additional file 1: Fig. 1a). Eighteen 35S:EsMYB90 transgenic tobacco and 15 35S: EsMYB90 transgenic Arabidopsis plants were obtained, respectively (Additional file 1: Fig. 1b,c).

We found that in all developing stages, leaves and stems in 35S:EsMYB90 tobacco plants appeared purplered, and the color became deepened with development (Fig. 3a-c). In addition, 35S:EsMYB90 tobacco plants produced purple-red corollas, purple-black sepals, and purple-black fruit pods, whereas wild-type corollas were pink, with green sepals and fruit pods (Fig. 3d-f). Our results from examining anthocyanin production showed that the total anthocyanin contents in three 35S:EsMYB90 tobacco lines were significantly increased in stems, young leaves (YL), mature leaves (ML), flowers, fruit pods, and mature seeds, compared with the wild type (Fig. 3g). Among L1, L2 and L4 three tested lines, the L4 transgenic line had the highest anthocyanin contents. Compared with the wild type, the total anthocyanin contents in young leaves (YL), mature leaves (ML), stems, flowers, fruits pods, and mature seeds of the L4 line were increased 95.2, 45.7, 48.8, 4.9, 17.8, and 2.6 folds, respectively (Fig. 3g). These results indicate that the enhanced pigmentation in 35S:EsMYB90 tobacco plants was caused by the increased synthesis of anthocyanins.

We observed similar phenotypes in 35S:EsMYB90 Arabidopsis transgenic plants. In comparison to the wild-type plants, the color of leaves, roots, stems, flowers, fruit pods, and seeds became light-purple to dark-purple in 35S: EsMYB90 Arabidopsis plants (Fig. 4a-g). In particularly, seeds from 35S:EsMYB90 Arabidopsis plants exhibited black color (Fig. 4h,i). Furthermore, the contents of anthocyanins in the roots, stems, leaves, flowers, and fruit pods at the bolting stage, and the mature seeds from three 35S:EsMYB90 Arabidopsis transgenic lines (L1, L2, and L3) were significantly higher than that in wild-type plants (Fig. 4j).

Collectively, our results suggest that EsMYB90 functions as a transcription factor to promote anthocyanin biosynthesis in plants.

\section{Transcriptomic analyses show that EsMYB90 is a key regulator in the proanthocyanidin and anthocyanin pathway}

To examine the molecular mechanisms by which EsMYB90 controls anthocyanin biosynthesis in the

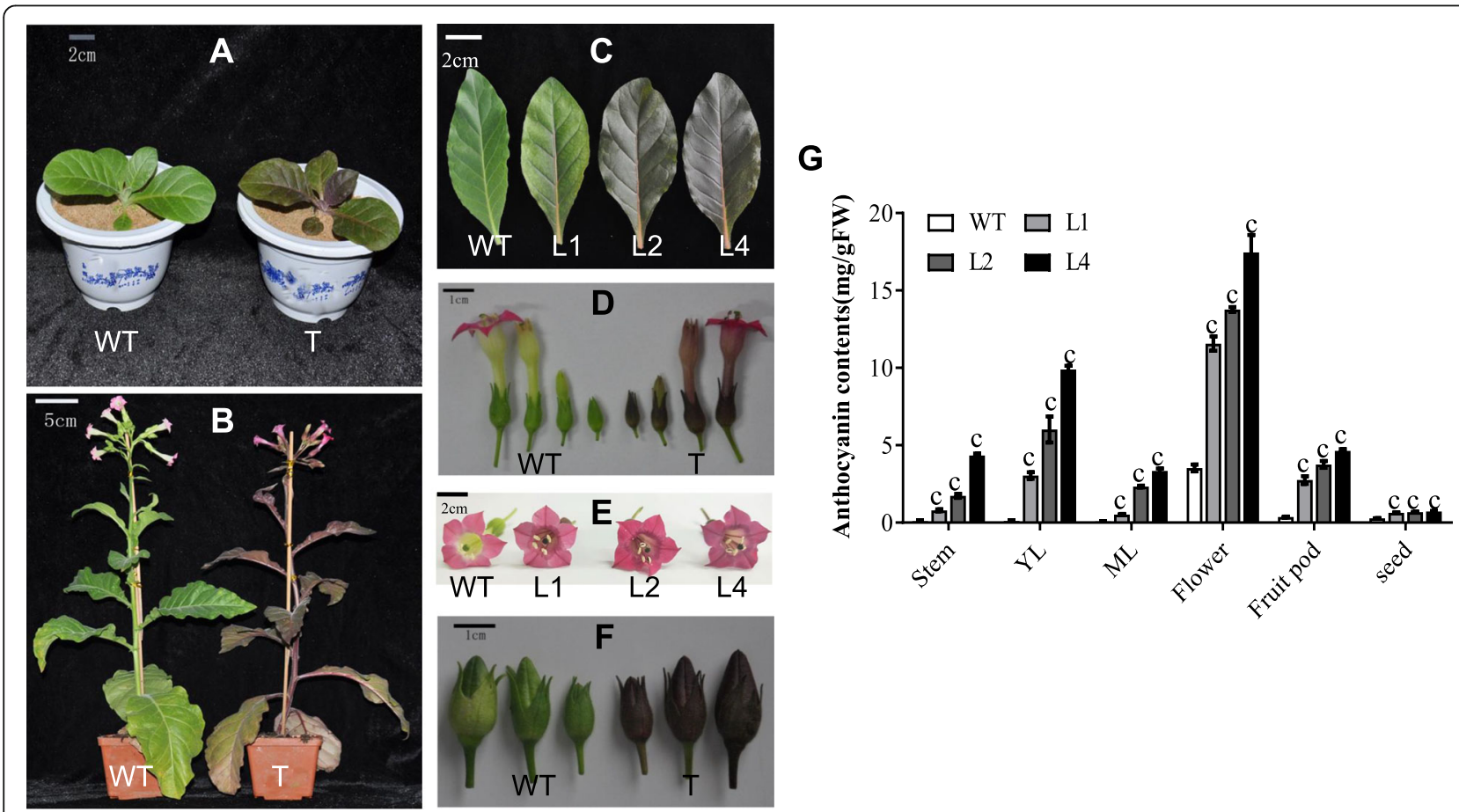

Fig. 3 Ectopic expression of ESMYB90 increases anthocyanin contents in tobacco. a Five-week-old wild-type (WT) and tobacco transgenic (T) seedlings; (b) Nine-week-old WT and tobacco T plants at the flowering stage; (c) Leaves from six-week-old WT as well as L1, L2 and L4 tobacco transgenic plants; (d) Sepal and corolla from nine-week-old WT and tobacco transgenic plants; (e) Flowers from nine-week-old WT as well as L1, $L 2$ and L4 tobacco transgenic plants; (f) Fruit pods from nine-week-old WT and tobacco transgenic plants; (g) Anthocyanin contents in stems, young leaves $(\mathrm{YL})$, mature leaves $(\mathrm{ML})$, flowers, fruit pods of eight-week-old plants, and mature seeds from WT as well as $L 1, L 2$ and $L 4$ tobacco transgenic plants. Vertical bars indicate standard errors of 3 biological replicates and the Student's t test values are indicated as c $(P<0.001)$ 


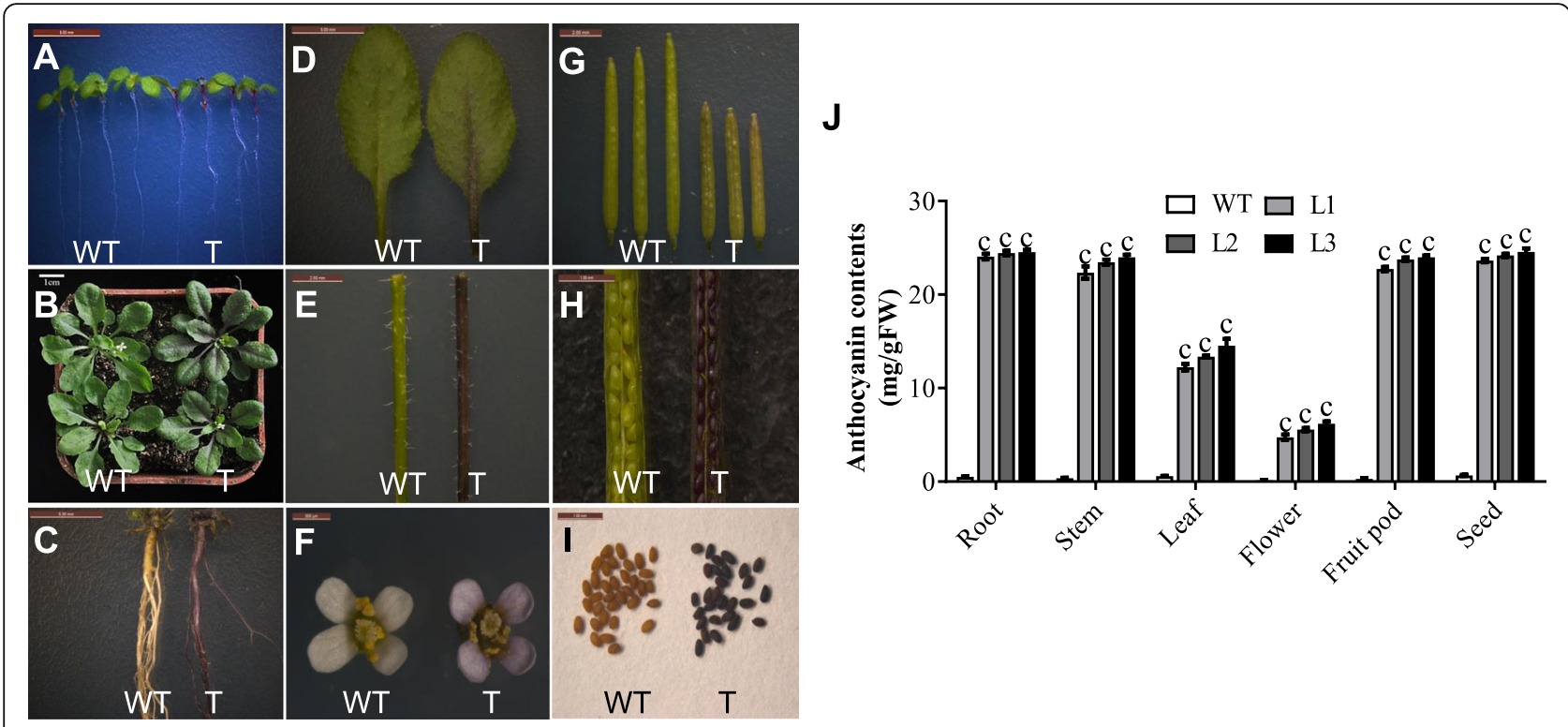

Fig. 4 Ectopic expression of ESMYB90 increases anthocyanin contents in Arabidopsis. (a) Seven-day-old wild-type (WT) and Arabidopsis transgenic (T) seedlings; (b) Three-week-old WT and Arabidopsis T plants; (c) Roots from seven-week-old WT and Arabidopsis T plants; (d) Leaves from three-week-old WT and Arabidopsis T plants; (e) Stems from seven-week-old WT and Arabidopsis T plants; (f) Flowers from seven-week-old WT and Arabidopsis T plants; (g) and (h) Fruit pods from seven-week-old WT and Arabidopsis T plants; (i) Seeds from nine-week-old WT and Arabidopsis T plants; (j) Anthocyanin contents in roots, stems, leaves, flowers, fruit pods of four-week-old plants and mature seeds, from WT as well as L1, L2 and L3 Arabidopsis transgenic plants. Vertical bars indicate standard errors of the 3 biological replicates and the Student's t test values are indicated as $c(p<0.001)$

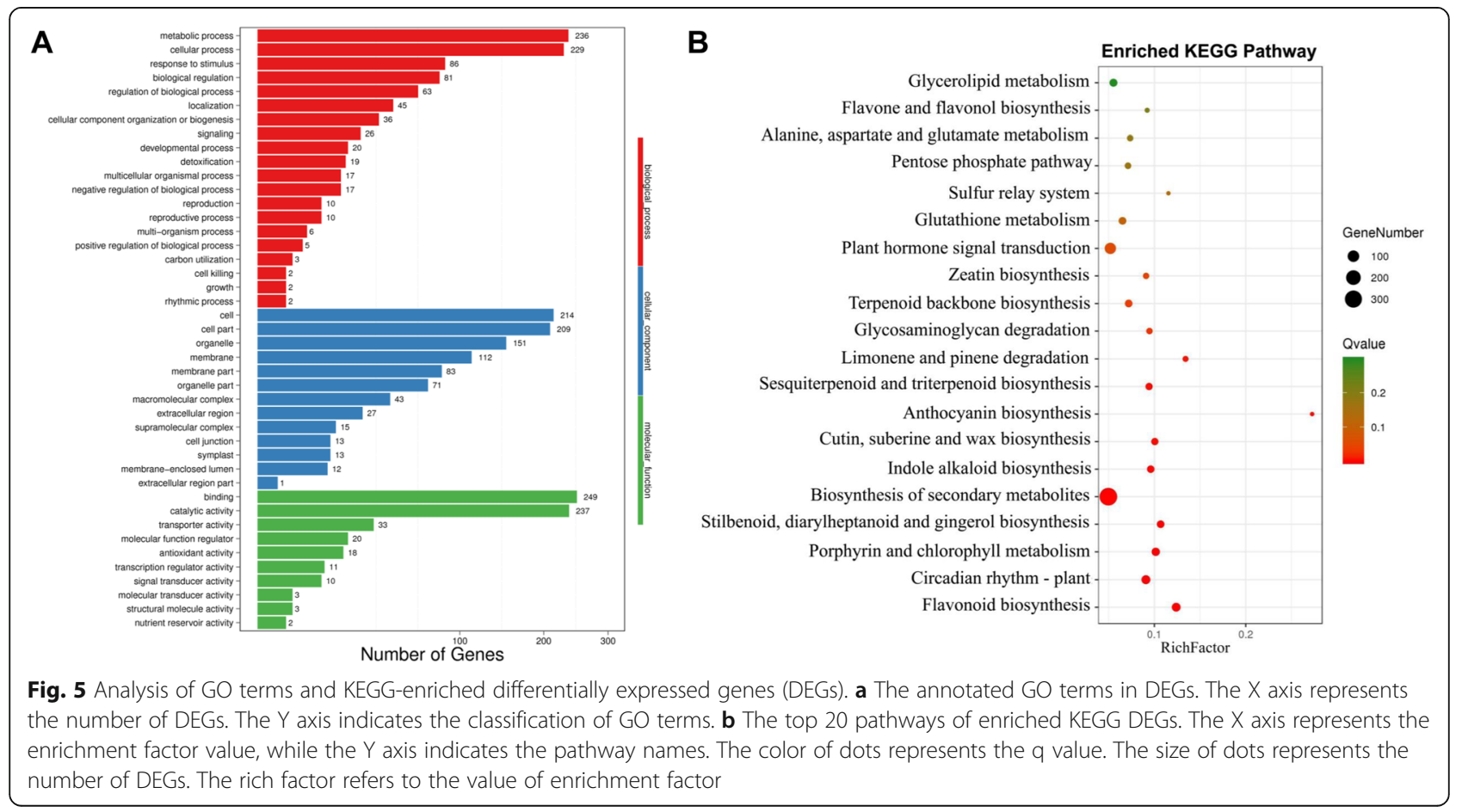


genome wide, we performed RNA-seq analysis using the leaves from wild-type and 35S:EsMYB90 tobacco transgenic plants. We identified 51,202 differentially expressed genes (DEGs) in the comparison of wild-type plant with 35S:EsMYB90 transgenic tobacco plants, among which 2446 DEGs have log2 Fold Change $\geq 1$ or $\leqq-1$ and a Padj $\leq 0.05$ (Additional file 2). Furthermore, 1199 out of 2446 DEGs were up-regulated, while 1247 genes were down-regulated (Additional file 2). Moreover, 476 unique DEGs were annotated into 43 GO terms, wherein the GO terms with the top 3 of the number of DEGs encoding the binding (249 genes), catalytic activities (237 genes) and metabolic processes (236 genes) (Fig. 5a, Additional file 3).

We revealed that among 2446 DEGs, 1023 unique genes were annotated into the 128 KEGG pathways (Additional file 4). The most prominent KEGG-enriched genes are involved in secondary metabolite biosyntheses, followed by plant hormone signaling, flavonoid biosynthesis (Additional file 1: Fig. 2). According to the q value of DEGs, the top 20 of enrichment paths included the flavonoid biosynthesis (ko00941), anthocyanin biosynthesis (ko00942), flavone and flavonol biosynthesis (ko00944), plant circadian rhythm (ko04712), and glutathione metabolism (ko00480;Fig. 5b, Additional file 5). Moreover, the anthocyanin biosynthesis pathway has the largest enrichment factor, followed by the pathway of flavonoid biosynthesis. The flavone and flavonol biosynthesis pathways also has a large enrichment factor (Fig. 5b, Additional file 5).

Mapping to the KEGG reference pathways found that a total of 57 significantly differential expression genes were assigned to five secondary metabolic pathways, i.e. phenylpropanoid biosynthesis (ko00940), flavonoid biosynthesis (ko00941), anthocyanin biosynthesis (ko00942), isoflavonoid biosynthesis (ko00943), and flavone and flavonol biosynthesis pathways (ko00944). The gene names, gene ID, and the combined functional annotations were seen in Additional file 6. Out of 57 genes, 42 genes encode PA and anthocyanin biosynthesis enzymes, such as PAL $(107,802,063,107,761,482$ and 107 , $820,497)$, CHS $(107,826,422,107,801,774$ and 107,813 , 613), CHI $(107,779,699,107,810,515$ and $107,825,576)$, F3H $(107,770,893,107,806,462)$, DFR $(107,803,097,107$, 797,232), and ANS/ LDOX (107,819,370, 107,778,118, $107,787,193,107,787,195$ and $107,808,500)$. Particularly, six genes encoding UFGT $(107,781,346,107,822,886$, $107,781,522,107,831,042,107,767,212$ and $107,819,220$ ) in the anthocyanin biosynthesis pathway (ko00942) were all strongly up-regulated (Fig. 6, Additional file 6). However, in ko00940-ko00944 pathways, only 15 genes including that encoding flavonol synthase/flavanone 3hydroxylase $(107,794,305,107,814,657)$, trans-resveratrol di-O-methyltransferase-like $(107,785,995,107,797,481)$, and flavone 3'-O-methyltransferase 1-like (107792977), were down-regulated (Fig. 6, Additional file 6).

Taken together, our RNA-seq results demonstrated that identified DEGs are significantly enriched in the flavonoid and anthocyanin synthesis pathway (ko00941ko00944), suggesting that EsMYB90 play an important regulatory role in proanthocyanidin and anthocyanin synthesis.

\section{Validation of RNA-seq results by qRT-PCR}

To validate the RNA-seq results, we performed quantitative reverse transcription PCR (qRT-PCR) for 18 genes which are assigned to 5 groups related to anthocyanin biosynthesis, antioxidant production, signal transduction, transcription regulation, and ion channel in tobacco (Additional file 7). Our results showed that expression level changes of 5 anthocyanin biosynthesis genes [NtDFR (107803097), NtLDOX54 (107778118), Nt3GT12 (107781346), Nt3GT36 (107781522), and Nt3GT53 (107831042)] detected by qRT-PCR were in agreement with the RNA-seq data (Fig. 7a). We obtained similar qRT-PCR results from examining expression of 4 antioxidant-related genes [NtP450 (107772738), NtCuZnSOD (107806960), NtPOD44-1 (107827231), and NtPOD44-2 (107797651); Fig. 7b], 4 genes encoding transcription factors [NtbZIP (107795590), NtMYB3R-1 (107795213), NtMYB4 (107802984), and NtWRKY53 (107825953); Fig. 7c], NtAKT2/3(107761230) encoding a potassium channel protein (Fig. $7 \mathrm{C}$ ), and 4 genes related to signal transduction and ion channel [NtMAPK3 (107782983), NtMAPK6 (107806359), NtAX15A (107805986), and NtCaM1 (107803626); Fig. 7d]. We found similar differential expression patterns for the DEGs in the qRT-PCR and RNA-seq data, with a lower pearson's coefficient (R2) as 0.9232. Therefore, qRTPCR results support that our transcriptome results are reliable.

\section{EsMYB90 promotes expression of anthocyanin biosynthetic genes in tobacco}

To further elucidate the molecular function of EsMYB90 in proanthocyanin and anthocyanin biosynthesis, we examined expression of key anthocyanin biosynthesis genes PAL, CHS, CHI, F3H, F3'H, DFR, ANS/LDOX, and UFGT in the stems, young leaves (YL) and flowers from 35S:EsMYB90 tobacco transgenic lines (L2, L4) and wild-type tobacco plants at the flowering stage by qRTPCR.

PAL is the first key enzyme in the metabolic pathway of phenylpropanoid [9]. Expression levels of NtPAL in stems, leaves and flowers from the L4 line increased 4.6, 7.1, and 2.8 times, respectively, than that of wild type (Fig. 8a). CHS catalyzes the first step of anthocyanin biosynthesis, while $\mathrm{CHI}$ catalyzes the cyclization of chalcone 


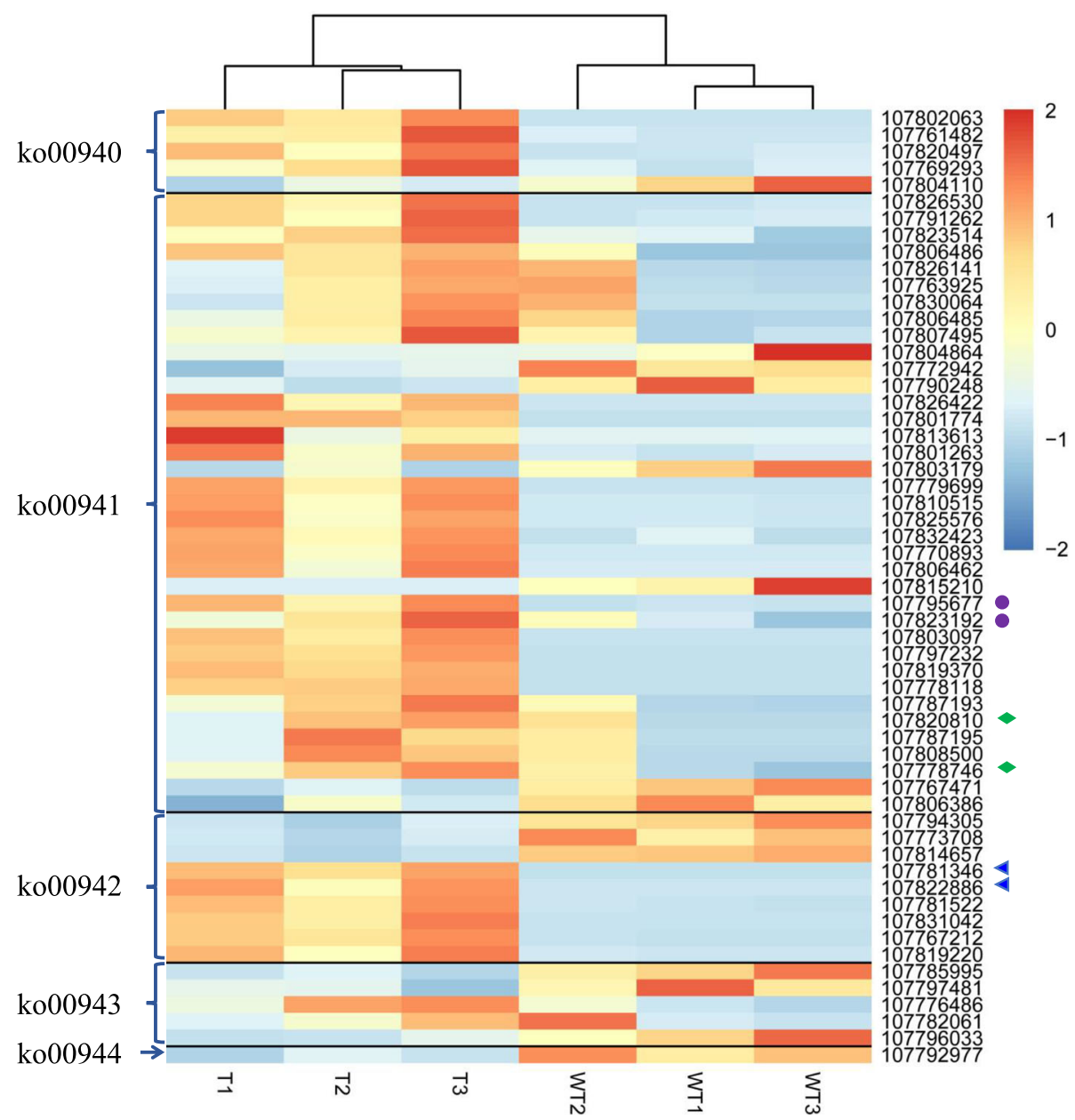

Fig. 6 Heatmap showing transcription levels of DEGs in flavonoid and anthocyanin synthesis pathways (ko00940-ko00944). Purple solid circles indicate 107,795,677 and 107,823,192 genes, which also exist in the ko00944 pathway. Green diamonds indicate 107,820,810 and 107,778,746 genes, which can be also found in the ko00942 pathway. Blue triangles indicate 107,781,346 and 107,822,886 genes, which co-exist in the ko00944 pathway

molecules to form naringenin [8]. Expression levels of both $N t C H S$ and $N t C H I$ in stems, leaves and flowers from the L4 line were significantly increased compared to that in the wild type (Fig. 8b,c). Whereas, the relative transcript level of NtF3H in the flowers was slightly down-regulated in $\mathrm{L} 4$ transgenic line (Fig. 8d), and $\mathrm{NtF}{ }^{\prime} \mathrm{H}$ transcripts in the stems were down-regulated in L2 and L4 transgenic lines (Fig. 8e). Finally, the anthocyanin biosynthesis genes NtDFR, NtANS and NtUFGT which are required for anthocyanin biosynthesis at later steps were also significantly upregulated by EsMYB90 (Fig. 8f-h).

In summary, our results suggest that EsMYB90 controls anthocyanin biosynthesis by promoting expression of anthocyanin biosynthesis genes, particularly LBGs.

\section{Discussion}

Anthocyanins are main contributors to the coloration of plants, and the color is an important determinant for fruit and flower quality $[8,14,56]$. Flavonoids such as anthocyanins are also capable of scavenging oxygen free radicals produced in cells, thus have antioxidant activity $[57,58]$. The antioxidant activity of anthocyanins depends on the degree of B-ring hydroxylation, acylation, and glycosylation [56]. Anthocyanins, as an antioxidant, can reduce the peroxidation of lipids and delay the aging of cells. Meanwhile, anthocyanins protect plants from damage caused by biotic and abiotic stresses and allow plants to adapt to environmental changes [59]. In higher plants, PAs and anthocyanin biosynthesis are regulated by different sets of MYB-bHLHWD40 (MBW) complexes, and the R2R3-MYBs play vital roles in transcriptional regulation of anthocyanins biosynthesis $[12,19,20,23,25,34,35]$. Our results shed light on the molecular mechanism by which a novel R2R3-MYB controls PA and anthocyanin biosynthesis via promoting expression of PA and anthocyanin biosynthesis genes. 


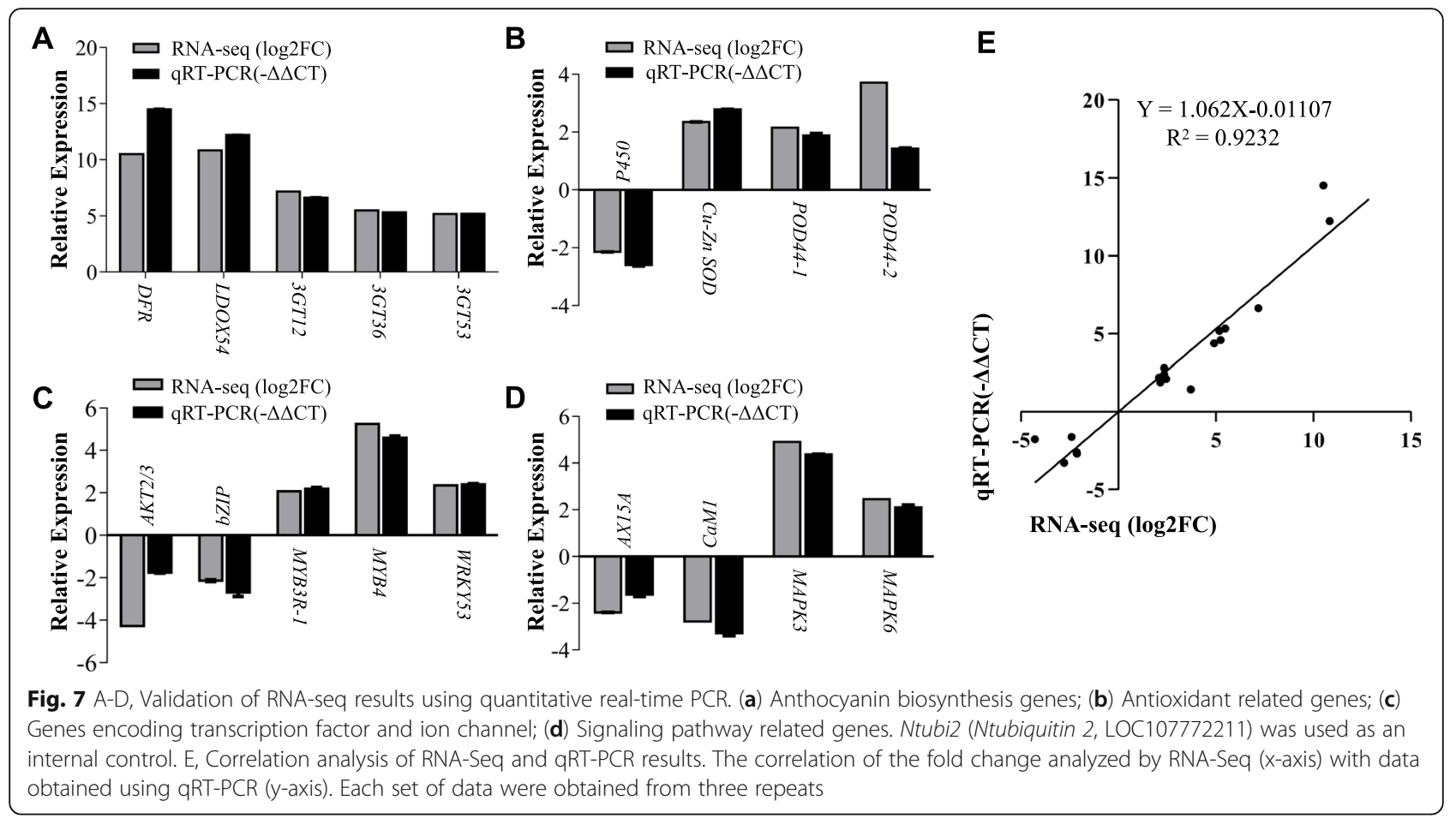

In Arabidopsis, MYB75, MYB90, MYB113, and MYB114 function redundantly by participating in the MBW (MYB-bHLH-WD40) complex to regulate PA and anthocyanin biosynthesis. Overexpression of one of these four MYBs is sufficient to increase anthocyanin accumulation in young leaves [15, 17]. EsMYB90 is a R2R3-MYB, containing a conserved [D/E]Lx2[R/ K]x3Lx6Lx3R motif (black arrows) required for interaction with R/B-like bHLH proteins, which was not found in EsMYB15 (XP_006406061), EsMYB106(XP_ 006390428), EsMYB108 (XP_006407939), EsMYB -related protein 340 (XP_006395259) from E. salsugineum. The ANDV (red box A) and KPRPR [S/T] F motifs (blue box B) characterizing anthocyanin-promoting MYBs exist in EsMYB90 protein but not in other five EsMYBs [MYB5 (XP_006407201), MYB15, MYB106, MYB108, MYB-related Protein 340] (Additional file 1: Fig. 3). In Arabidopsis and some other higher plants, the anthocyanin pathway is controlled by multiple MYB transcriptional factors [1]. For instance, at least four MBW complexes assembled with various MYBs are involved in the PA accumulation in the innermost cell layer of Arabidopsis seed coat [23]. Thus, our results suggest that different from other plants less MYBs regulate anthocyanin biosynthesis in E. salsugineum and EsMYB90 is a major player.

The regulatory mechanisms for flavonoid biosynthesis are conserved in higher plants [1]. The MYB proteins usually determine the involvement of MBW complexes in specific pathways $[1,60]$. In the MBW complex,
MYBs have the highest binding specificity compared with bHLH and WD40. MYB and bHLH bind to at least one of three conserved cis elements, i.e. MYB-core, ACrich, and E/G-box, in promoters of four LBGs (late biosynthesis genes: DFR, TT19, TT12, and AHA10), which specify their expression in the seed coat of Arabidopsis $[23,60]$. Expression of LBGs are regulated by MYB75, MYB90, MYB113 and MYB114 in Arabidopsis [15, 16]. Our phylogenetic analysis showed that EsMYB90 was in the same clade with Arabidopsis MYB75, MYB90, MYB113, and MYB114, suggesting that EsMYB90 may play a more important role in regulation of LBGs (DFR, $A N S / L D O X$ and UFGT) expressions, which is in accordance with our research results. In addition, our results show that EsMYB90 promotes expression of early biosynthesis genes, such as PAL, CHS, CHI, and F3H which agrees with findiCngs in other plants $[1,10,36]$. Furthermore, it is possible that EsMYB90 upregulates expressions of $N t M Y B$ and $N t b H L H$ genes involved in anthocyanin biosynthesis, because expressions of NtMYB3 (107820930), NtMYB4 (107,802,984, 107,769,018, 107,815, 562, 107,760,435), NtMYB44 (107,816,351, 107,759,750), MYB-like (107,807,565, 107,795,213), NtbHLH (107,791, 671, 107,791,200), NtbHLH92 (107785534), NtbHLH18 (107811232), and NtbHLH35(107774314) were significantly increased in 35S:EsMYB90 tobacco transgenic plants (Additional file 2).

Overall, our results showed that ectopic expression of the novel EsMYB90 gene can strongly induce the anthocyanin biosynthesis by promoting expression of 


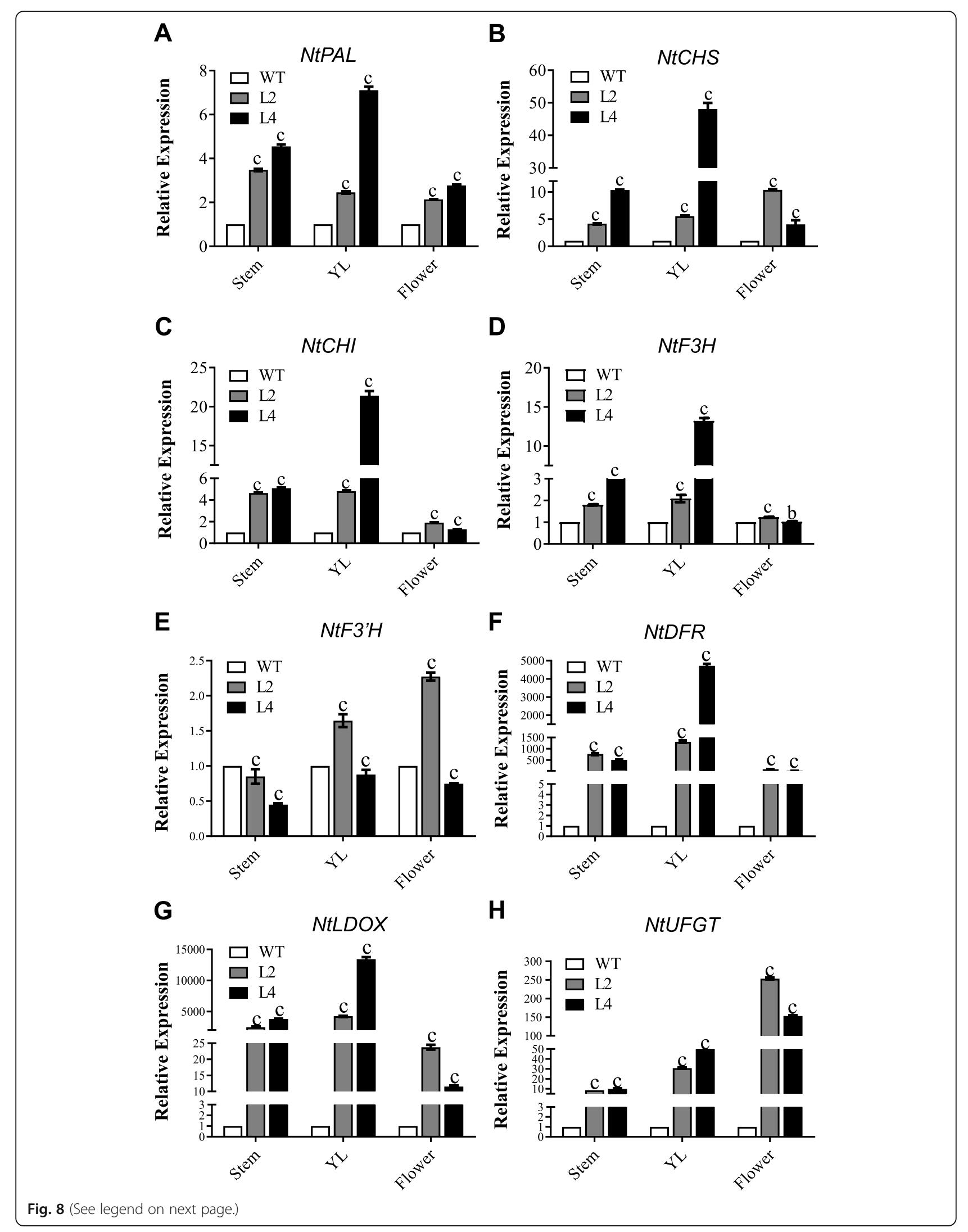


(See figure on previous page.)

Fig. 8 Expression analysis of anthocyanin biosynthesis genes in stem, leaf and flower from two EsMYB90 transgenic tobacco lines at the flowering stage. WT: Wild Type; L2 and L4: two independent 35S:EsMYB90 lines. Ntubi2 (Ntubiquitin 2, LOC107772211) was used as an internal control. (a) NtPAL (XM_016625506.1); (b) NtCHS (NM_001326166.1); (c) NtCHI (NM_001325287.1); (d) NtF3H (NM_001325083.1); (e) NtF3'H (NM_001325608.1); (f) NtDFR (NM_001325732.1); (g) NtLDOX (NM_001326043.1); (h) NtUFGT (NM_001325312.1). The $\Delta \Delta$ Ct method was used to determine the relative expression, and expression levels of tested genes in the wild type were set to 1. Vertical bars represent standard errors of three biological replicates. $b$ and $c$ indicate $p<0.01$ and $p<0.001$, respectively

anthocyanin biosynthesis pathway genes, particularly functioning in the LBGs. Our study also paved the way for the application of EsMYB90 to genetically engineering crops and ornamental plants.

\section{Conclusions}

EsMYB90, a R2R3 MYB transcription factor, is localized in nucleus. 35S:EsMYB90 transgenic tobacco and Arabidopsis showed purple-red, purple-black phenotypes, and accumulated more anthocyanin in the leaves, stems and flowers compared with wild type. The results showed that ectopic expression of EsMYB90 in stems, leaves and flowers of transgenic tobacco could significantly enhanced the expression of anthocyanin biosynthetic genes including EBGs (PAL, CHS, and CHI) and LBGs (DFR, $A N S$, and UFGT), particularly in LBGs. The study suggested that EsMYB9O plays a key role in regulating anthocyanin biosynthesis, and it provide new clues to increase the content of anthocyanin in transgenic plants.

\section{Methods}

\section{Plant materials and growth conditions}

The seeds of Eutrema salsugineum (Shandong ecotype), Arabidopsis thaliana (Columbia-0) and tobacco (Nicotiana tabacum cv SR1) are preserved and presented by Shandong Provincial Key Laboratory of Plant Stress Research, College of Life Science, Shandong Normal University.

Wild-type and transgenic plants of Nicotiana tabacum and Arabidopsis thaliana (Columbia-0) were grown in a mixture of vermiculite, perlite and peat moss (1:1:1) in a greenhouse with $25^{\circ} \mathrm{C}$, a photoperiod of $16 \mathrm{~h}$ light $/ 8 \mathrm{~h}$ dark. Eutrema salsugineum were grown in a growth chamber with $22^{\circ} \mathrm{C}$, a photoperiod of $16 \mathrm{~h}$ light $/ 8 \mathrm{~h}$ dark and $70 \%$ relative humidity.

T3 generation homozygous transgenic and wild-type tobacco plants were used for analyses. After 8 weeks growth of tobacco plants at the period of 8-9 leaves, the sixth leaves of wild-type and transgenic plants were collected for transcriptome and GRT-PCR identification of the transcriptome. Three biological repeats for transcriptome and qRT-PCR were performed. In tobacco, stems, young leaves $(\mathrm{YL})$, mature leaves $(\mathrm{ML})$, flowers, and fruit pods at the flowering stage, and mature seeds were used to determine the content of anthocyanins, meanwhile the stems, young leaves (YL), and flowers were used to examine expression of anthocyanin biosynthesis genes. Similarly, Arabidopsis roots, stems, leaves, flowers, and fruit pods from plants growing 4 weeks, and mature seeds were used to determine the content of anthocyanins. In all cases, samples were frozen immediately in liquid nitrogen and stored at $-80^{\circ} \mathrm{C}$. Three repeats of all tests were conducted.

\section{Phylogenetic analysis}

A set of associated MYB protein sequences were downloaded from the NCBI and multiple sequence alignments were carried out using the DNAMAN software (Version 5.2.2). The phylogenetic tree was constructed using the MEGA6 software with the neighbor-joining method.

\section{Subcellular localization of EsMYB90}

The coding region of EsMYB90 was PCR-amplified by YFPMYB-F and YFPMYB-R primers with SacI and SacII sites, respectively (Additional file 9) and then cloned in to the YFP-pCAT vector, resulting in the 35S:YFPEsMYB90- $p C A T$ vector. The onion epidermal cells were transformed with the 35S:YFP-EsMYB90-pCAT and YFP- $p C A T$ (control) plasmids, respectively, using the plasmid bombardment method [61]. The transformed cells were incubated at $25^{\circ} \mathrm{C}$ for $16-24 \mathrm{~h}$, then the florescence signals were observed and recorded with a fluorescence microscope (BX51, model 7.3, Olympus, Tokyo, Japan). At least three replicates for each construct were performed.

\section{RNA isolation and CDNA synthesis}

Total RNAs were isolated from $0.5 \mathrm{~g}$ of young leaves of Eutrema salsugineum and Nicotiana tabacum using the TRIzol Reagent (Life Technologies) or Quick RNA isolation kit (Biotech biotechnology company, Beijing, China). The RNA concentration and purity(A260/A280) were measured with a NanoDrop ND1000 spectrophotometer (NanoDrop Technologies, Wilmington, DE, USA). First-strand cDNA was synthesized using the Rever Tra Ace qPCR RT Master Mix with gDNA Remover (Toyobo, Japan). Briefly, RNA was heat-denatured at $65^{\circ} \mathrm{C}$ for $5 \mathrm{~min}$ and immediately placed on the ice for cooling, then $2 \mu \mathrm{L}$ of $4 \times \mathrm{DN}$ Master Mix, $0.5 \mathrm{pg}-0.5 \mu \mathrm{g}$ of RNA template and nuclease-free water were added to $8 \mu \mathrm{L}$ of volume. After $5 \mathrm{~min}$ of incubation at $37^{\circ}, 5 \times \mathrm{RT}$ Mater Mix II was added to make $10 \mu \mathrm{L}$ of final volume. 
RT reaction was carried out at $37^{\circ} \mathrm{C}$ for $15 \mathrm{~min}, 50{ }^{\circ} \mathrm{C}$ for $5 \mathrm{~min}, 98^{\circ} \mathrm{C}$ for $5 \mathrm{~min}$ to obtain $10 \mu \mathrm{l}$ of cDNA.

\section{Construction generation and plant transformation} The whole coding sequence of EsMYB9O was PCRamplified from the Eutrema salsugineum cDNA using the forward primer 5'-CCGGAATTCTTTAGAATAC TTATTGGTCC-3' and the reverse primer 5 '-CGCGGA TCCATCAGAGACAGATATTAGTTGG-3' with EcoR I and BamH I restriction enzyme sites at the $5^{\prime}$ and $3^{\prime}$, respectively (Additional file 9). The resulting EsMYB90 fragment was cloned into the pMD18-T vector (Takara, USA). After sequencing confirmation, the EsMYB90 fragment was subcloned into the EcoRI - BamHI sites of the pCAMBIA3301H vector, where EsMYB90 was under the control of the $C a M V 35 S$ promoter. The expression vector (35S:EsMYB90-pCAMBIA3301H) was finally introduced into the Agrobacterium tumefaciens strain GV3101.

Transformation of $N$. tabacum was performed using the leaf disc method essentially as reported by Horsch et al. [62]. Transgenic tobacco seedlings were selected on the MS medium containing $6 \mathrm{mg} / \mathrm{L}$ of bastar and $300 \mathrm{mg} / \mathrm{L}$ of cefalexin. Transformation of A. thaliana Columbia-0 was performed using the floral-dipping method [63] and transformants were screened by spraying $0.1 \%$ of bastar herbicide. The presence of the transgene was further confirmed by PCR using specific primers for EsMYB90. The homozygous transgenic $N$. tabacum and $A$. thaliana were used for subsequent phenotypic and functional analysis.

\section{Anthocyanin analysis}

Stems, young leaves $(\mathrm{YL})$, mature leaves $(\mathrm{ML})$, flowers, and fruit pods of tobacco growing about 8 weeks at the flowering stage, and the mature seeds were sampled, respectively. Similarly, stems, leaves, flowers, fruit pods, and roots of Arabidopsis growing about 4 weeks at the bolting period, as well as the mature seeds were also collected. All materials were frozen immediately in liquid nitrogen and ground to powders. The anthocyanin content was determined using an improved method described by Neff and Chory [64]. The measurements of $A_{530}$ and $A_{657}$ were conducted with a spectrophotometer (UV-1800, Shimadzu). The results were calculated by the eq. (A530-0.25*A657)/fresh weight. Three replicates were performed for each sample.

\section{RNA-seq and bioinformatic analysis}

Total RNAs from the sixth leaves of wild-type and EsMYB90 transgenic tobacco at the 7-8 leaves stage were isolated using a Quick RNA isolation kit (Bioteke Corporation, Beijing, China). The RNA library construction and sequencing were performed in the BGI
Corporation (Shenzhen, China) using the BGISEQ-500 platform. Three independent biological replicates were carried out.

The low-quality reads (more than 20\% of the bases qualities are lower than 10), reads with adaptors and reads with unknown bases ( $\mathrm{N}$ bases more than $5 \%$ ) were filtered to get the clean reads. The clean reads were mapped to the reference genome using HISAT [65]. Meanwhile, the clean reads were mapped to the reference transcripts using Bowtie2 [66]. The clean reads were assembled into unigenes, followed by the unigene functional annotation, etc., and calculate the unigene expression levels of each sample [67]. Finally, we identified DEGs (differential expressed genes) and performed clustering analysis and functional annotations. DEGs with the GO and KEGG annotation results were classified according to the official classification, and the GO and KEGG pathway functional enrichment were performed using phyper in the $\mathrm{R}$ software. Transcription Factor Prediction of DEG: The ORF of each DEG were founded using getorf and aligned to TF domains (from PlntfDB) using hmmsearch [68].

\section{Gene expression analysis by qRT-PCR}

To validate the transcriptome results, the real-time qPCR was performed by the LightCycler 96 system to examine expressions of selected genes (Additional file 9) using the total RNAs extracted from tobacco leaves used for RNA-seq. Transcriptome data was verified by comparing the result of qRT-PCR $(-\Delta \Delta \mathrm{CT})$ with RNA-seq $(\log 2 \mathrm{FC})$.

To examine expression of anthocyanin biosynthesis genes in different tissues, stems, young leaves $(\mathrm{YL})$ and flowers of tobacco at the flowering stage were sampled, respectively. The total RNAs were extracted using the total RNA rapid extraction kit (Biotech biotechnology company, China). The first-strand cDNAs were synthesized using the Rever Tra Ace qPCR RT Master Mix with gDNA Remover (Toyobo, Japan). The qPCR was performed using the LightCycler 96 system (Roche, Switzerland; Supplementary Table S1). Ntubi2 (ubiquitin 2, LOC107772211) was used as an internal reference gene. Three replicates were performed for each sample.

\section{Supplementary information}

Supplementary information accompanies this paper at https://doi.org/10. 1186/s12870-020-02391-7.

Additional file 1. Fig. 1. Molecular identification of ESMYB90 transgenic tobacco and Arabidopsis plants. Fig. 2. Pathways of the most enriched KEGG DEGs in RNA-Seq of ESMYB90 transgenic tobacco. Fig. 3. Sequence alignment analysis of the EsMYB90 and other EsMYB proteins in E.salsugineum. 
Additional file 2. Differentially expressed genes (DEGs) that meet log2 Fold Change $\geq 1$ or $\leq-1$ and a Padj $\leq 0.05$ in RNA-Seq of EsMYB90 transgenic tobacco.

Additional file 3. Annotated DEGs in GO terms in RNA-Seq of EsMYB9O transgenic tobacco.

Additional file 4. Annotated DEGs in KEGG in RNA-Seq of EsMYB9O transgenic tobacco.

Additional file 5. Top 20 paths according to the RichFactor value of DEGs.

Additional file 6. DEGs enriched in the flavonoid and anthocyanin synthesis pathways.

Additional file 7. Detailed data for validation of RNA-Seq results using quantitative real-time PCR

Additional file 8. Detailed data for the expression analysis of anthocyanin biosynthesis genes in stem, young leaf and flower from two ESMYB90 transgenic tobacco lines and WT.

Additional file 9. Sequences of the primers used in the study.

\section{Abbreviations}

PAs: Proanthocyanidins; PAL: Phenylalanine ammonia-lyase; CHS: Chalcone synthase; CHI: Chalcone isomerase; DHK: Dihydrokaempferol\; F3H: Flavanone $3 \beta$-hydroxylase; DHQ: Dihydroquercetin; F3'H: Flavonoid 3'-hydroxylase; DHM: Dihydromyricetin; F3'5'H: Flavonoid 3',5'-hydroxylase.;

DFR: Dihydroflavonol 4-reductase; ANS: Anthocyanidin synthase; UFGT: UDPglucoseflavonoid 3-O-glucosyltranferase; MBW: MYB-bHLH-WD40; EBGs: Early biosynthesis genes; LBGs: Late biosynthesis genes; YL: Young leaves; ML: Mature leaves; DEGs: Differentially expressed genes; KEGG: Kyoto Encyclopedia of Genes and Genomes; GO: Gene Ontoloty; qRT-

PCR: Quantitative reverse transcription PCR

\section{Acknowledgments}

We thank the anonymous reviewers for their valuable comments and suggestions.

\section{Authors' contributions}

QZ conceived and designed the experiments. QZ wrote the manuscript with contributions from CM, XW, SG, and CG, CG, YQ, SG,CL1, CZ, and CL2 carried out most of the experiments. CL1 and CZ took care of the plants. QZ,YQ,CG and CL2 analyzed the data. All authors read and approved the final manuscript.

\section{Funding}

This study was supported by the National Major Science and Technology Project of China (2018ZX08009-10B), Key Technology Research and Development Program of Shandong (2019GSF107089), and Natural Science Foundation of Shandong Province (ZR2014CM041). The funding body has no role in the design of the study and collection, analysis, and interpretation of data and in writing the manuscript.

\section{Availability of data and materials}

All data generated or analyzed during this study are included in this published article and its additional files. The raw data of RNA library were available at NCBI Short Read Archive (PRJNA609528).

\section{Ethics approval and consent to participate}

Not applicable.

\section{Consent for publication}

Not applicable.

\section{Competing interests}

The authors declare that they have no competing interests.

\section{Author details}

'Shandong Provincial Key Laboratory of Plant Stress Research, College of Life Science, Shandong Normal University, Jinan 250014, China. 'Biotechnology Research Center, Shandong Academy of Agricultural Sciences, Shandong Provincial Key Laboratory of Crop Genetic Improvement, Ecology and
Physiology, Jinan 250100, China. ${ }^{3}$ Beijing Engineering Research Center for Hybrid Wheat, The Municipal Key Laboratory of the Molecular Genetics of Hybrid Wheat, Beijing Academy of Agriculture and Forestry Sciences, Beijing 100097, China

Received: 27 November 2019 Accepted: 12 April 2020

Published online: 28 April 2020

\section{References}

1. Xu WJ, Dubos C, Lepiniec L. Transcriptional control of flavonoid biosynthesis by MYB-bHLH-WDR complexes. Trends Plant Sci. 2015;20(3):176-85.

2. Lou Q, Liu YL, Qi YY, Jiao SZ, Tian FF, Jiang L, Wang YJ. Transcriptome sequencing and metabolite analysis reveals the role of delphinidin metabolism in flower colour in Grape hyacinth. J Exp Bot. 2014;65(12):3157-64.

3. Falcone Ferreyra ML, Rius SP, Casati P. Flavonoids: biosynthesis, biological functions and biotechnological applications. Front Plant Sci. 2012;3(222):222.

4. Hichri I, Barrieu F, Bogs J, Kappel C, Delrot S, Lauvergeat V. Recent advances in the transcriptional regulation of the flavonoid biosynthetic pathway. J Exp Bot. 2011;62(8):2465-83.

5. Zhang Y, Butelli E, Martin C. Engineering anthocyanin biosynthesis in plants. Curr Opin Plant Biol. 2014;19:81-90.

6. Niu SS, Xu CJ, Zhang WS, Zhang B, Li X, Lin-Wang K, Ferguson IB, Allan AC, Chen KS. Coordinated regulation of anthocyanin biosynthesis in Chinese bayberry (Myrica rubra) fruit by a R2R3 MYB transcription factor. Planta. 2010;231(4):887-99.

7. Petroni $\mathrm{K}$, Tonelli $\mathrm{C}$. Recent advances on the regulation of anthocyanin synthesis in reproductive organs. Plant Sci. 2011;181(3):219-29.

8. Jaakola L. New insights into the regulation of anthocyanin biosynthesis in fruits. Trends Plant Sci. 2013;18(9):477-83.

9. Jones $\mathrm{DH}$. Phenylalanine ammonia-lyase: regulation of its induction, and its role in plant development. Phytochem. 1984;23(7):1349-59.

10. Liu XF, Xiang LL, Yin XR, Grierson D, Li F, Chen KS. The identification of a MYB transcription factor controlling anthocyanin biosynthesis regulation in Chrysanthemum flowers. Sci Hortic. 2015;194:278-85.

11. Takos AM, Jaffé FW, Jacob SR, Bogs J, Robinson SP, Walker AR. Lightinduced expression of a MYB gene regulates anthocyanin biosynthesis in red apples. Plant Physiol. 2006;142(3):1216-32.

12. Pérez-Díaz JR, Pérez-Díaz J, Madrid-Espinoza J, González-Villanueva E, Moreno Y, Ruiz-Lara S. New member of the R2R3-MYB transcription factors family in grapevine suppresses the anthocyanin accumulation in the flowers of transgenic tobacco. Plant Mol Biol. 2016;90(1-2):63-76.

13. Feng SQ, Wang $Y L$, Yang $S, X u Y T$, Chen XS. Anthocyanin biosynthesis in pears is regulated by a R2R3-MYB transcription factor PyMYB10. Planta. 2010:232(1):245-55.

14. Zhou H, Peng Q, Zhao JB, Owiti A, Ren F, Liao L, Wang L, Deng XB, Jiang Q, Han YP. Multiple R2R3-MYB transcription factors involved in the regulation of anthocyanin accumulation in peach flower. Front Plant Sci. 2016;7(28): 1557

15. Gonzalez A, Zhao M, Leavitt JM, Lloyd AM. Regulation of the anthocyanin biosynthetic pathway by the $T \mathrm{G} 1 / \mathrm{bHLH} /$ Myb transcriptional complex in Arabidopsis seedlings. Plant J. 2008;53(5):814-27.

16. Zimmermann IM, Heim MA, Weisshaar B, Uhrig JF. Comprehensive identification of Arabidopsis thaliana MYB transcription factors interacting with R/B-like bHLH proteins. Plant J. 2004;40(1):22-34.

17. Bac-Molenaar JA, Fradin EF, Rienstra JA, Vreugdenhil D, Keurentjes JJ. GWA mapping of anthocyanin accumulation reveals balancing selection of MYB90 in Arabidopsis thaliana. PLoS One. 2015:10(11):e0143212

18. Zuluaga DL, Gonzali S, Loreti E, Pucciariello C, Degl'Innocenti E, Guidi L, Alpi A, Perata P. Arabidopsis thaliana MYB75/PAP1 transcription factor induces anthocyanin production in transgenic tomato plants. Funct Plant Biol. 2008; 35(7):606-18.

19. Borevitz JO, Xia Y, Blount J, Dixon RA, Lamb C. Activation agging identifies a conserved MYB regulator of phenylpropanoid biosynthesis. Plant Cell. 2000; 12:2383-93.

20. Tohge T, Nishiyama Y, Hirai MY, Yano M, Nakajima J, Awazuhara M, Inoue E, Takahashi H, Goodenowe DB, Kitayama M, Noji M, Yamazaki M, Saito K. Functional genomics by integrated analysis of metabolome and ranscriptome of Arabidopsis plants over-expressing an MYB transcription factor. Plant J. 2005;42(2):218-35

21. Ilk N, Ding J, Ihnatowicz A, Koornneef M, Reymond M. Natural variation for anthocyanin accumulation under high light and low temperature stress is 
attributable to the enhancer of AG-42 (hua2) locus in combination with production of anthocyanin pigment1 (PAP1) and PAP2. New Phytol. 2015; 206(1):422-35.

22. Lepiniec L, Debeaujon I, Routaboul JM, Baudry A, Pourcel L, Nesi N, Caboche M. Genetics and biochemistry of seed flavonoids. Annu Rev Plant Biol. 2006;57(1):405-30.

23. Xu WJ, Grain D, Bobet S, Le Gourrierec J, Thévenin J, Kelemen Z, Lepiniec L, Dubos C. Complexity and robustness of the flavonoid transcriptional regulatory network revealed by comprehensive analyses of MYB-bHLH-WDR complexes and their targets in Arabidopsis seed. New Phytol. 2014;202(1): $132-44$.

24. Nesi N, Jond C, Debeaujon I, Caboche M, Lepiniec L. The Arabidopsis $\pi 2$ gene encodes an R2R3 MYB domain protein that acts as a key determinant for proanthocyanidin accumulation in developing seed. Plant Cell. 2001; 13(9):2099-114.

25. Stracke R, Werber M, Weisshaar B. The R2R3-MYB gene family in Arabidopsis thaliana. Curr Opin Plant Biol. 2001;4(5):447-56.

26. Chiu LW, Zhou X, Burke S, Wu X, Prior RL, Li L. (2010). The purple cauliflower arises from activation of a MYB transcription factor. Plant Physiol. 2013; 154(3):1470-80.

27. Zhang Y, Chen G, Dong T, Pan Y, Zhao Z, Tian S, Hu Z. Anthocyanin accumulation and transcriptional regulationof anthocyanin biosynthesis in purple Bok Choy (Brassicarapa var. chinensis). J Agric Food Chem. 2013; 62(51):12366-76

28. Peng T, Moriguchi T. The molecular network regulating the coloration in apple. Sci Hortic. 2013;163:1-9.

29. Telias A, Lin-Wang K, Stevenson DE, Cooney JM, Hellens RP, Allan AC, Hoover EE, Bradeen JM. Apple skin patterning is associated with differential expression of MYB10. BMC Plant Biol. 2011;11(1):93.

30. Chagné D, Carlisle CM, Blond C, Volz RK, Whitworth CJ, Oraguzie NC, Crowhurst RN, Allan AC, Espley RV, Hellens RP, Gardiner SE. Mapping a candidate gene (MdMYB10) for red flesh and foliage colour in apple. BMC Genomics. 2007;8(1):212.

31. Rahim MA, Busatto N, Trainotti L. Regulation of anthocyanin biosynthesis in peach fruits. Planta. 2014;240(5):913-29.

32. Bai S, Tao R, Tang Y, Yin L, Ma Y, Ni J, Yan X, Yang Q, Wu Z, Zeng Y, Teng Y. BBX16, a B-box protein, positively regulates light-induced anthocyanin accumulation by activating MYB10 in red pear. Plant Biotechnol J. 2019;17: 1985-97.

33. Qian M, Sun Y, Allan AC, Teng Y, Zhang D. The red sport of 'Zaosu' pear and its red-striped pigmentation pattern are associated with demethylation of the PyMYB10 promoter. Phytochemistry. 2014;107:16-23.

34. Medina-Puche L, Cumplido-Laso G, Amil-Ruiz F, Hoffmann T, Ring L, Rodríguez-Franco A, Caballero JL, Schwab W, Muñoz-Blanco J, BlancoPortales R. MYB10 plays a major role in the regulation of flavonoid/ phenylpropanoid metabolism during ripening of Fragaria $x$ ananassa fruits. J Exp Bot. 2014;65(2):401-17.

35. Schwinn K, Venail J, Shang Y, Mackay S, Alm V, Butelli E, Oyama R, Bailey P, Davies K, Martin C. A small family of myb-regulatory genes controls floral pigmentation intensity and patterning in the genus Antirrhinum. Plant Cell. 2006;18(4):831-51.

36. Chen KL, Liu HL, Lou Q, Liu YL. Ectopic expression of the grape hyacinth (Muscari armeniacum) R2R3-MYB transcription factor gene, MaAN2, induces anthocyanin accumulation in tobacco. Front Plant Sci. 2017:8:965.

37. Terrier $\mathrm{N}$, Torregrosa $\mathrm{L}$, Ageorges $A$, Vialet $\mathrm{S}$, Verriès $\mathrm{C}$, Cheynier $\mathrm{V}$, Romieu $\mathrm{C}$. Ectopic expression of VvMybPA2 promotes proanthocyanidin biosynthesis in grapevine and suggests additional targets in the pathway. Plant Physiol. 2009;149(2):1028-41.

38. Cavallini E, Zenoni S, Finezzo L, Guzzo F, Zamboni A, Avesani L, Tornielli GB. Functional diversification of grapevine MYB5a and MYB5b in the control of flavonoid biosynthesis in a petunia anthocyanin regulatory mutant. Plant Cell Physiol. 2014;55(3):517-34.

39. Liu XF, Feng C, Feng C, Zhang MM, Yin XR, Xu CJ, Chen KS. The MrWD40-1 gene of Chinese bayberry (Myrica rubra) interacts with MYB and bHLH to enhance anthocyanin accumulation. Plant Mol Biol Rep. 2013;31(6):1474-84.

40. Huang WJ, Sun W, Lv HY, Luo M, Zeng SH, Pattanaik S, Yuan L, Wang Y. A R2R3-MYB transcription factor from Epimedium sagittatum regulates the flavonoid biosynthetic pathway. PLoS One. 2013;8(8):e70778.

41. Huang WJ, LV HY, Wang Y. Functional characterization of a novel R2R3-MYB transcription factor modulating the flavonoid biosynthetic pathway from Epimedium sagittatum. Front Plant Sci. 2017;8:1274.
42. Mellway RD, Tran LT, Prouse MB, Campbell MM, Constabel CP. The wound-, pathogen-, and ultraviolet B-responsive MYB134 gene encodes an R2R3 MYB transcription factor that regulates proanthocyanidin synthesis in poplar. Plant Physiol. 2009;150(2):924-41.

43. Liu Y, Lin-Wang K, Espley RV, Wang L, Yang H, Yu B, Dare A, Varkonyi-Gasic E, Wang J, Zhang J, Wang D, Allan AC. Functional diversification of the potato R2R3 MYB anthocyanin activators AN1, MYBA1, and MYB113 and their interaction with basic helix-loop-helix cofactors. J Exp Bot. 2016;67(8): 2159-76.

44. Oh DH, Leidi E, Zhang Q, Hwang SM, Li YZ, Quintero FJ, Jiang XY, D'Urzo MP, Lee SY, Zhao YX, Bahk JD, Bressan RA, Yun DJ, Pardo JM, Bohnert HJ. Loss of halophytism by interference with SOS1 expression. Plant Physiol. 2009:151(11):210-22.

45. Inan G, Zhang Q, Li PH, Wang ZL, Cao ZY, Zhang H, Zhang CQ, Quist TM, Goodwin SM, Zhu JH, Shi HZ, Damsz B, Charbaji T, Gong QQ, Ma SS, Fredricksen M, Galbraith DW, Jenks MA, Rhodes D, Hasegawa PM, Bohnert HJ, Joly RJ, Bressan RA, Zhu JK. Salt cress. A halophyte and cryophyte Arabidopsis relative model system and its applicability to molecular genetic analyses of growth and development of extremophiles. Plant Physiol. 2004; 135(3):1718-37.

46. Wang JS, Zhang Q, Cui F, Hou L, Zhao SZ, Xia H, Qiu JJ, Li TT, Zhang Y, Wang XJ, Zhao CZ. Genome-wide analysis of gene expression provides new insights into cold responses in Thellungiella salsuginea. Front Plant Sci. 2017; 8:713.

47. Ali Z, Park HC, Ali A, Oh DH, Aman R, Kropornicka A, Hong H, Choi W, Chung WS, Kim WY, Bressan RA, Bohnert HJ, Lee SY, Yun DJ. TsHKT1;2, a HKT1 homolog from the extremophile Arabidopsis relative Thellungiella salsuginea, shows $\mathrm{K}^{+}$specificity in the presence of $\mathrm{NaCl}$. Plant Physiol. 2012; 158(3):1463-74.

48. Sun W, Li Y, Zhao YX, Zhang H. The TsnsLTP4, a nonspecific lipid transfer protein involved in wax deposition and stress tolerance. Plant Mol Biol Rep. 2015;33(4):962-74.

49. Zhang Q, Zhao CZ, Li M, Sun W, Liu Y, Xia H, Sun MN, Li AQ, Li CS, Zhao SZ, Hou L, Picimbon JF, Wang XJ, Zhao YX. Genome-wide identification of Thellungiella salsuginea microRNAs with putative roles in the salt stress response. BMC Plant Biol. 2013;13(1):180.

50. Shamloo-Dashtpagerdi R, Razi H, Ebrahimie E, Niazi A. Molecular characterization of Brassica napus stress related transcription factors, BnMYB44 and BnVIP1, selected based on comparative analysis of Arabidopsis thaliana and Eutrema salsugineum transcriptomes. Mol Biol Rep. 2018;45(5):1111-24

51. Zhu L, Guo J, Zhu J, Zhou C. Enhanced expression of EsWAX1 improves drought tolerance with increased accumulation of cuticular wax and ascorbic acid in transgenic Arabidopsis. Plant Physiol Biochem. 2014;75:24-35.

52. Griffith M, Timonin M, Wong AC, Gray GR, Akhter SR, Saldanha M, Rogers MA, Weretilnyk EA, Moffatt B. Thellungiella: an arabidopsis-related model plant adapted to cold temperatures. Plant Cell Environ. 2007;30(5):529-38.

53. Dubos C, Stracke R, Grotewold E, Weisshaar B, Martin C, Lepiniec L. MYB transcription factors in Arabidopsis. Trends Plant Sci. 2010;15(10):573-81.

54. Quattrocchio F, Wing JF, van der Woude K, Mol JN, Koes R. Analysis of $\mathrm{bHLH}$ and MYB domain proteins: species-specific regulatory differences are caused by divergent evolution of target anthocyanin genes. Plant J. 1998; 13(4):475-88

55. Shirley BW, Kubasek WL, Storz G, Bruggemann E, Koornneef M, Ausubel FM, Goodman HM. Analysis of Arabidopsis mutants deficient in flavonoid biosynthesis. Plant J. 1995;8(5):659-71.

56. Sadilova E, Stintzing FC, Carle R. Anthocyanins, colour and antioxidant properties of eggplant (Solanum melongena L.) and violet pepper (Capsicum annuum L.) peel extracts. Z Naturforsch C. 2006;61(7-8):527-35.

57. Hernández I, Alegre L, Van Breusegem F, Munné-Bosch S. How relevant are flavonoids as antioxidants in plants? Trends Plant Sci. 2009;14(3):125-32.

58. Nakabayashi R, Yonekura-Sakakibara K, Urano K, Suzuki M, Yamada Y, Nishizawa T, Matsuda F, Kojima M, Sakakibara H, Shinozaki K, Michael AJ, Tohge T, Yamazaki M, Saito K. Enhancement of oxidative and drought tolerance in Arabidopsis by overaccumulation of antioxidant flavonoids. Plant J. 2014;77(3):367-79.

59. Ahmed NU, Park Jl, Jung HJ, Yang TJ, Hur Y, Nou IS. Characterization of dihydroflavonol 4-reductase (DFR) genes and their association with cold and freezing stress in Brassica rapa. Gene. 2014;550(1):46-55.

60. Heppel SC, Jaffé FW, Takos AM, Schellmann S, Rausch T, Walker AR, Bogs J. Identification of key amino acids for the evolution of promoter target 
specificity of anthocyanin and proanthocyanidin regulating MYB factors. Plant Mol Biol. 2013;82(4-5):457-71.

61. Kinkema M, Fan WH, Dong XN. Nuclear localization of NPR1 is required for activation of $P R$ gene expression. Plant Cell. 2000;12(12):2339-50.

62. Horsch R, Fry JE, Hoffmann NL, et al. A simple and general method for transferring genes into plants. Science. 1985;227:1229-32.

63. Clough SJ, Bent AF. Floral dip : a simplified method for Agrobacteriummediated transformation of Arabidopsis thaliana. Plant J. 1998;16(6):735-43.

64. Neff MM, Chory J. Genetic interactions between phytochrome a, phytochrome B, and cryptochrome 1during Arabidopsis development. Plant Physiol 1998;118(1):27-36.

65. Kim D, Langmead B, Salzberg SL. HISAT: a fast spliced aligner with low memory requirements. Nat Meth. 2015;12(4):357-60

66. Langmead B, Salzberg SL. Fast gapped-read alignment with bowtie 2. Nat Meth. 2012:9(4):357-9.

67. Li B, Dewey CN. RSEM: accurate transcript quantification from RNA-Seq data with or without a reference genome. BMC Bioinformatics. 2011;12(1):323.

68. Mistry J, Finn RD, Eddy SR, Bateman A, Punta M. Challenges in homology search: HMMER3 and convergent evolution of coiled-coil regions. Nucleic Acids Res. 2013;41(12):e121.

\section{Publisher's Note}

Springer Nature remains neutral with regard to jurisdictional claims in published maps and institutional affiliations.

Ready to submit your research? Choose BMC and benefit from:

- fast, convenient online submission

- thorough peer review by experienced researchers in your field

- rapid publication on acceptance

- support for research data, including large and complex data types

- gold Open Access which fosters wider collaboration and increased citations

- maximum visibility for your research: over $100 \mathrm{M}$ website views per year

At $\mathrm{BMC}$, research is always in progress.

Learn more biomedcentral.com/submissions 